\title{
Hydrogen Adsorption Studies Using Surface Acoustic Waves on Nanoparticles
}

\author{
A.B. Phillips ${ }^{1}$, G. Myneni ${ }^{2}$, B.S. Shivaram ${ }^{1}$ \\ ${ }^{1}$ Department of Physics, University of Virginia, Charlottesville, Virginia. 22901. \\ 2 Jefferson Labs, Newport News, Virginia.
}

\begin{abstract}
Nanoparticles made from metals such as Vanadium and Niobium, on the order of $10 \mathrm{~nm}$, are deposited on a quartz surface acoustic wave resonator (SAWR) using a Nd:YAG pulsed laser deposition system. Utilizing the high Q and resonant frequency of the SAWR, mass changes due to adsorption on the order of 0.1 nanogram can be measured. Thus, this technique enables the controlled collection of isolated nanoparticles with total mass at the $10 \mathrm{~s}$ of nanogram level and subsequent sorption of hydrogen in them. At room temperature and 4.9 bar of hydrogen pressure, we find $1.75 \mathrm{wt} \% \mathrm{H}$ absorption ratio in $\mathrm{V}$ nanoparticles of $10 \mathrm{~nm}$ nominal size.
\end{abstract}

Keywords: nanoparticle, hydrogen storage, SAW

PACS: 61.46. $+\mathrm{w}$, 66.30.Pa, 81.16.Mk, 64.75. $+\mathrm{g}, 65.80 .+\mathrm{n}$

\section{INTRODUCTION}

Hydrogen adsorption studies in nanoparticles are both of fundamental and practical interest[1-4]. While many techniques exist to measure hydrogen adsorption[5,6] in bulk samples, there are few techniques to measure isolated nanoparticles and novel nanostructures rapidly being invented and assembled today[7]. Surface acoustic wave resonators, with high Q and resonant frequency, allow for such measurements to be made[8,9]. Furthermore, with isolated nanoparticles it is possible to form novel material compositions that do not exist in the bulk[10]. Hydrogen adsorption studies in such "compositionally challenged" alloys should also be of interest.

\section{EXPERIMENT}

\section{Surface Acoustic Wave Resonators}

A surface acoustic wave resonator (SAWR) is a piezoelectric crystal with a set of interdigital transducers (IDT), which excite the crystal to form standing waves confined to the surface and an additional IDT to sample the excited wave. Perturbations of the velocity $\mathrm{v}$ and amplitude $\alpha$ of the surface waves occur under several conditions. Mass loading by film or nanoparticles deposition is one such 


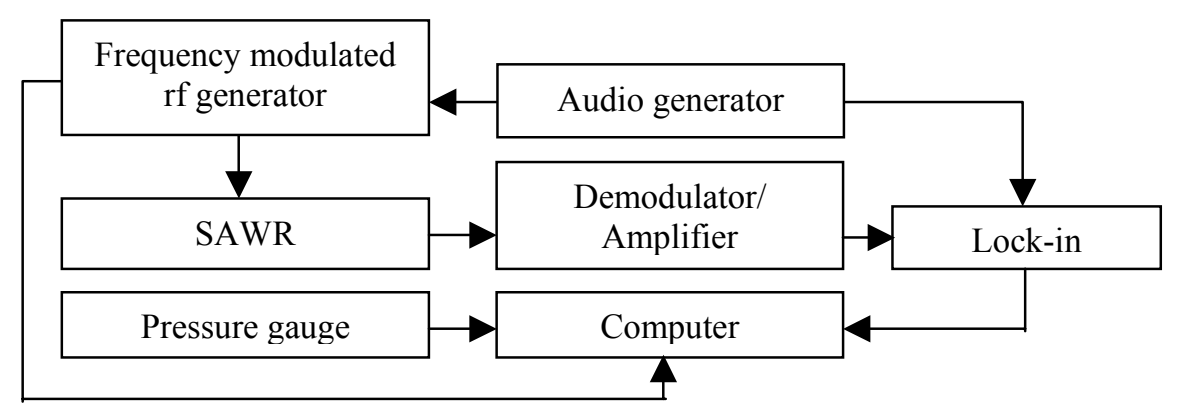

FIGURE 1. A rf signal is frequency modulated with a low frequency signal before exciting the sample. The response is then demodulated and measured using a lock-in amplifier.

mechanism. Additionally, when the number of nanoparticles deposited is relatively small or when the film is acoustically thin a readily measurable change in $\mathrm{v}$ can occur without a significant enhancement in $\alpha$. By incorporating the SAWR in an oscillatory circuit, the fractional frequency change, $\frac{\Delta f}{f_{0}}$ (equal to the fractional velocity change), can be related to the mass loading of the resonator through the equation:

$$
\frac{\Delta f}{f_{0}}=\frac{\Delta v}{v_{0}}=-c_{m} f_{0} \frac{\Delta m}{A}
$$

Here the mass sensitivity factor $\mathrm{c}_{\mathrm{m}}$ is a substrate dependant parameter (for quartz $\mathrm{c}_{\mathrm{m}}=1.3 \mathrm{~cm}^{2} / \mathrm{g} \cdot \mathrm{MHz}$ ) and $\mathrm{A}$ is the resonant cavity area[7].

In our experiments we use an rf frequency SAWR made by RF Monolithics, RP 1239 , which resonates at $315 \mathrm{MHz}$. A frequency modulation technique where the $\mathrm{rf}$ signal is modulated at audio low frequencies is employed. The modulated rf excitation signal connects to one port of the SAWR and the sampling port of the SAWR connects to a wideband amplifier and a demodulator. Figure 1 shows a diagram of the measurement setup. To determine the resonant frequency, the rf

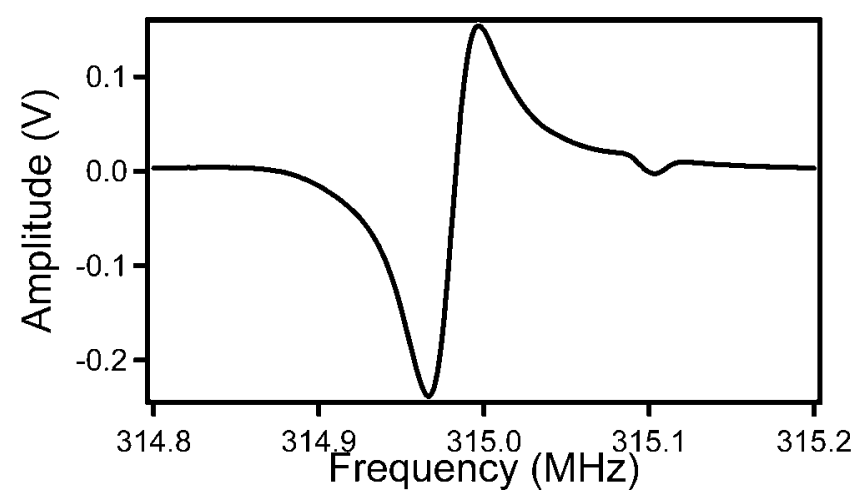

FIGURE 2. The bare SAWR response as frequency is scanned is shown. The resonant frequency is where the signal crosses zero, in this case $314.969 \mathrm{MHz}$. 
signal is varied and the amplitude of the SAWR response recorded. A typical response is shown in Fig. 2.

\section{Nanoparticle Deposition}

Nanoparticles of the requisite metal are deposited directly onto the SAWR by pulsed laser deposition. A Nd:YAG laser with $8 \mathrm{~ns}$ pulse and repetition rate of $10 \mathrm{~Hz}$ is used to ablate material from the target. A frequency doubled beam obtained by passing the fundamental of the Nd:YAG laser through a harmonic generator and separator with wavelength of $532 \mathrm{~nm}$ is employed in the ablation process. Using a Molectron PowerMAX 500D, the power of the beam was measured to be $200 \mathrm{~mW}$. The chamber is backfilled with Ar to $500 \mathrm{mTorr}$ so the particles form in flight and deposit on the room temperature SAWR substrate. The deposited mass of nanoparticles is continuously monitored by tracking the resonant frequency of the SAWR. Due to the rapidity of the deposition process, in practice we found that it is simpler to hold the excitation frequency of the SAWR constant and measure the response. As the mass increases, the previously measured response shifts to a lower frequency, as indicated by the negative sign in Equation (1).

\section{Hydrogen Loading}

Upon completion of deposition, the SAWR with the nanoparticles and a reference SAWR with no deposited material are moved to a high-pressure hydrogen loading cell. During the hydrogen loading experiments a zero tracking method is employed to measure the resonant frequency. In this method the zero crossing of the SAWR response is first measured by sweeping the rf frequency about the original resonance. When the response changes sign, the zero crossing frequency, and thus the resonant frequency, is recalculated in real time and a new excitation frequency is set on the $\mathrm{rf}$ generator. The resonant frequencies of the SAWR with the sample and the reference SAWR are measured as a function of pressure as hydrogen gas is continuously leaked into the chamber until the final pressure of 23.4 bar is reached. The same measurements are repeated as the hydrogen is removed from the cell back to the original pressure of 0.83 bar.

\section{RESULTS}

We estimate the total V deposited on the SAWR as $50.4 \mathrm{ng}$. We also measure the size and distribution of the particles using a transmission electron microscope (TEM). For consistent deposition parameters, the TEM grid used to collect the nanoparticles is mounted on the same SAWR holder prior to ablation. Figure 3 is a picture of the particles as observed through TEM. The average particle size under the conditions listed above is $10 \mathrm{~nm}$. 


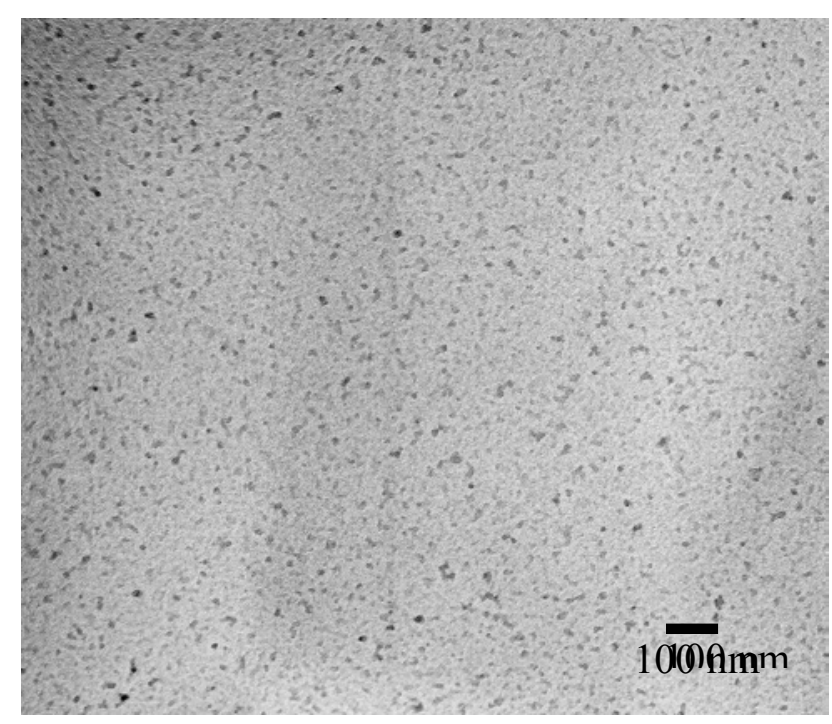

FIGURE 3. The TEM is used to investigate the size and distribution of $\mathrm{V}$ nanoparticles, which formed in flight while under $500 \mathrm{mTorr}$ Ar pressure. The average size of the particles is $10 \mathrm{~nm}$.

The high-pressure measurements of the sample show the saturation point for the V samples in terms of both pressure and mass loading. Figure 4 shows the hydrogen percentage mass loading of the $\mathrm{V}$ nanoparticles as well as the pressure. The total mass of the hydrogen adsorbed by the vanadium is $0.880 \mathrm{ng}$, or $1.75 \mathrm{wt}$ $\%$. The saturation of the vanadium samples occurs at 4.9 bar. The sample also releases all of the adsorbed hydrogen upon returning to the original pressure.

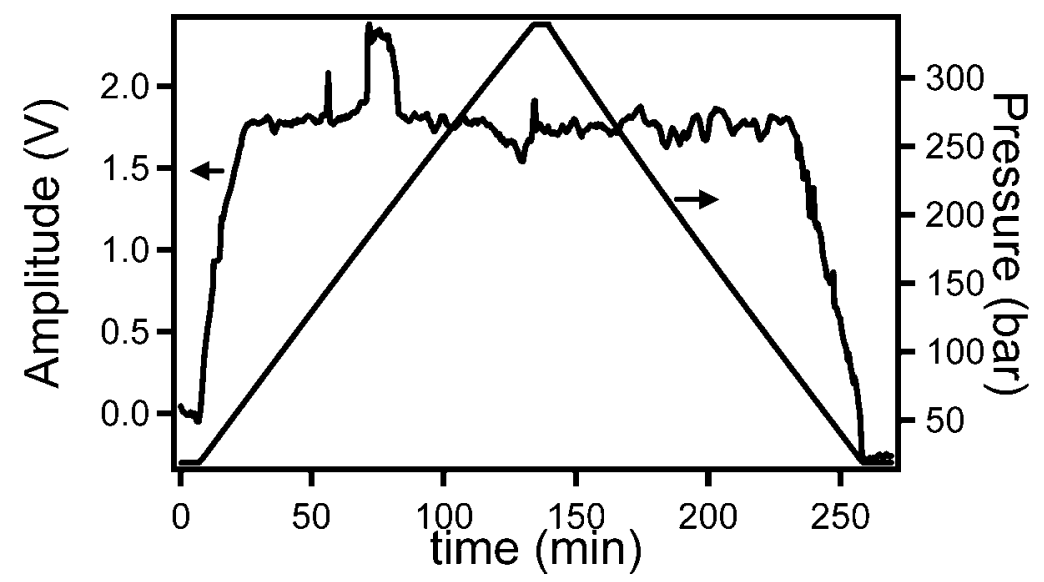

FIGURE 4. The mass loading of the SAWR and the pressure are measured as a function of time. At saturation hydrogen is $1.75 \mathrm{wt} \%$ of the vanadium particles. The saturation occurs at a pressure of 4.9 bar. 


\section{SUMMARY}

We have shown that pulsed laser deposition can be used to create freestanding nanoparticles of $\mathrm{Nb}$ and $\mathrm{V}$ of controlled size. Additionally, by using a SAWR as the substrate, we can measure the mass of the particles deposited. We have also shown that a SAWR is sensitive enough to measure hydrogen sorption onto nanoparticles on the order of picograms.

\section{ACKNOWLEDGMENTS}

Partial support for this project is provided by the National Science Foundation through DMR-0073456. Support also comes from Jefferson Lab managed by Southeastern University Research Association for the United States Department of Energy Office of Science. We would also like to thank Tom Schamp for his help with TEM microscopy and analysis.

\section{REFERENCES}

1. C.C. Koch, J.D. Whittenberger, Intermetallics 4 339-355 (1996).

2. T. Mütshele, R. Kirchheim, Scripta Metallurgica 21 1101-1104 (1987).

3. N. Hanada, T. Ichikawa, S.-I. Orimoc, H. Fujii, J Alloys Compounds 366 269-273 (2004).

4. J.A. Eastman, L.J. Thompson, B.J. Kestel, Phys Rev B 48 84-92 (1993).

5. J. Huot, J.F. Pelletier, G. Liang, M. Sutton, R. Schulz, J Alloys Compounds 330-332 727731 (2002).

6. R. Janot, A. Rougier, L. Aymard, C. Lenain, R. Herrera-Urbina, G.A. Nazri , J.M. Tarascon, J Alloys Compounds 356-357 438-441 (2003).

7. T. Ono, X. Li, H. Miyashita, M. Esashi, Rev Sci Instr 74 1240-1243 (2003).

8. D.S. Ballantine, R.M. White, S.J. Martin, A.J. Ricco, E.T. Zellers, G.C. Frye, H. Wohltjen, Acoustic Wave Sensors: Theory, Design, and Physio-Chemical Applications, Academic Press, New York, NY, 1997.

9. V.I. Anisimkin, E. Verona, IEEE Transactions on Ultrasonics, Ferroelectrics, and Frequency Control 45 1347-1354 (1998).

10. W.A. Jesser, G.J. Shiflet, G.L. Allen, J.L. Crawford, Mat Res Innovat 2 211-216 (1999). 